\title{
Applications of metabolomics to the environmental sciences
}

\author{
Mark R. Viant
}

Published online: 18 February 2009

(C) Springer Science+Business Media, LLC 2009

Metabolomics has become a versatile technique that is widely used by academia and industry in the medical, toxicological, nutritional, and other biological sciences. The purpose of focusing this journal issue on "Environmental Metabolomics" is to both recognize and highlight the growing number of metabolomics studies within the environmental sciences. Some may regard environmental metabolomics purely as the application of this technique to study organisms dwelling within the natural environment, with the goal to learn about the state or condition of the environment. However, the field is in fact considerably broader than this, including the application of metabolomics to areas such as ecophysiology - with a focus on understanding the underpinning biochemical responses of organisms to abiotic and biotic stressors in their environment; and to ecotoxicology and ecological risk assessmentwhich typically involves chemical toxicity testing within a controlled laboratory environment to inform upon the potential risks of chemicals to organisms in the natural environment. Ultimately all environmental metabolomics studies probe organism-environment interactions with the goal to characterise organism function at the molecular level and/or to inform upon environmental health.

A review by Bundy et al. provides a comprehensive overview of environmental metabolomics studies to date, including a critical evaluation of the contribution that metabolomics has made to the environmental sciences. In addition, the authors discuss a number of recommendations to advance this field. As highlighted in this review, some of the pioneering work in environmental metabolomics

M. R. Viant ( $\square)$

School of Biosciences, University of Birmingham, Edgbaston, Birmingham B15 2TT, UK

e-mail: M.Viant@bham.ac.uk included characterising the responses of organisms to toxic stress, with the potential of discovering novel biomarkers for subsequent environmental diagnostics. This is still a highly active field of study, evidenced by the six original articles in this special issue. Ekman et al. report the sex and time dependent metabolic responses of the fathead minnow (Pimephales promelas), a sentinel test species, to the synthetic estrogen 17-ethynylestradiol. The novel aspect of this research is the focus on the lipid metabolome, which has received minimal attention in environmental metabolomics. The ability of NMR metabolomics to differentiate responses to pesticides (atrazine and lindane) and natural environmental stressors (hypoxia and starvation), which is a prerequisite for its application in ecological monitoring, is highlighted by Greenwood et al. in studies of the marine mussel Mytilus edulis. Taylor and colleagues describe the first application of metabolomics to toxicity testing in Daphnia magna, a water flea that is used internationally for environmental risk assessment. They utilised direct infusion Fourier transform ion cyclotron resonance (FT-ICR) mass spectrometry to identify multiple metabolic responses to copper toxicity, and putatively identified more than a thousand low molecular weight polar metabolites in the tissue extracts. A time course study into the toxicity of the herbicide prometryn on a unicellular green alga (Scenedesmus vacuolatus) is reported by Kluender et al. The authors concluded that GC-MS studies of synchronous algal cultures represent a valuable new tool in ecotoxicology.

Metabolomics studies of earthworms have been ongoing for more than a decade. Here three further studies are reported which serve to strengthen the growing literature on the applicability of earthworms for metabolomics-based environmental monitoring and chemical risk assessment. Using NMR spectroscopy, Guo and colleagues have confirmed that a diverse range of toxicants with unique modes 
of action (cadmium, atrazine and fluoranthene) induce specific and distinct metabolic responses in the earthworm Lumbricus rubellus. McKelvie et al. report the metabolic responses of Eisenia fetida to two pesticides, DDT and endosulfan, using both NMR and GC/MS based approaches. In addition, Rochfort and co-workers studied two species of earthworm, an unidentified native Australian species and Aporrectodea caliginosa, which were sampled directly from the field to inform on land treatment regimes. Land-use was shown to have a marked effect on the biochemistry of the worm populations. A related article that uses metabolomics to investigate processes in soil is described by Fan and colleagues who utilised ${ }^{13} \mathrm{C}$ isotopomer-based methods to study multiple microbial groups isolated from temperate and tropical soils in order to track microbial metabolism.

The three final original articles in this issue highlight the diversity of environmental metabolomics. Karakach et al. utilised both NMR and LC-MS based approaches to investigate the molecular mechanisms associated with chronic stress in juvenile Atlantic salmon (Salmo salar), which is important both within an ecological context and in aquaculture. The authors report a number of new potential markers of stress from their time course study. The effects of environmental stress, specifically atmospheric temperature, on the metabolism of Arabidopsis lyrata ssp. petraea is discussed by Davey et al. This investigation determined that plant populations sampled from different latitudes (and therefore varying minimal temperatures) exhibit differences in their metabolic phenotypes, suggesting adaptation to the local thermal environment. Finally, biotic-biotic interactions are the subject of the study by Jansen et al., who investigated changes in the metabolomes of both the feral cabbage (Brassica oleracea) and small cabbage white caterpillar (Pieris rapae) in response to shoot and root induction. The authors conclude that the metabolomics approach is useful for discovering bioactive compounds involved in ecological interactions.

In summary, this special issue is intended to highlight both the growing interest in applying metabolomic techniques to the environmental sciences, and the diversity of the nature of such studies. The eleven original articles reported here include studies on two fish and two aquatic invertebrate species' (including marine and freshwater), five terrestrial invertebrate species (of which four are earthworms), three plant species, and multiple microbial strains. The applications focus upon ecotoxicology, chemical risk assessment and biomarker discovery, tools for assessing ecological and soil health, and investigations into the effects of other biotic and abiotic environmental stressors. The value of metabolomics to these fields is now being recognised by environmental scientists, both academics as well as "end-users" involved in regulation and monitoring. This is clearly evidenced by funding within the US and UK to provide dedicated environmental metabolomics facilities, e.g. by the US EPA and UK NERC. Such facilities should enable the continued growth of this vibrant field. I hope you enjoy these articles and any comments can be sent to me at the address above. 